\title{
Wavefront Sensor for the ESA-GAIA Mission
}

\author{
L.L.A. Vosteen*, Draaisma F.,Werkhoven, W.P., Riel L.J.M.., Mol, M.H., Ouden G. den \\ TNO Science and Industry, Stieltjesweg 1,2600 AD Delft, The Netherlands
}

\begin{abstract}
TNO developed a Wave Front Sensor (WFS) instrument for the GAIA mission. This Wave Front Sensor will be used to monitor the wave front errors of the two main telescopes mounted on the GAIA satellite, which may be corrected by a 5degree of freedom (DOF) mechanism during operation. The GAIA-WFS will operate over a broad wavelength (450 to $900 \mathrm{~nm}$ ) and under cryogenic conditions (130 to $200 \mathrm{~K}$ operation temperature). The WFS uses an all reflective, a-thermal design and is of the type of Shack-Hartmann. The boundary condition for the design is that the focal plane of the WFS is the same plane as the focal plane of the GAIA telescopes. The spot pattern generated after a micro lens array ( MLA) by a star is compared to the pattern of one of the three calibration sources that is included in the WFS, allowing in flight calibration. We show the robust and lightweight opto mechanical design that is optimised for launch and cryogenic operation. Furthermore we give details on its alignment and commissioning. The WFS can measure wave front distortions in the order of lambda/1000, and determines the focal plane with an accuracy of $50 \mu \mathrm{m}$
\end{abstract}

Keywords: Cryogenic, Shack Hartmann, Wave front sensor, Opto mechanical, INVAR, exoplanets

\section{INTRODUCTION}

The ESA Gaia mission is a successor to the ESA Hipparcos mission. It's objective is to create the largest and most precise three dimensional chart of our galaxy by providing unprecedented positional and radial velocity measurements for about one billion stars in our Galaxy and throughout the local group. Each of its target stars will be monitored about 100 times over 5 year, precisely charting their distances, movements and changes in brightness. It is expected to discover hundreds of thousands of new celestial objects, such as exoplanets and failed stars, GAIA should also identify tens of thousands of asteroids.

Gaia is being built by EADS Astrium and is scheduled for launch end 2011. This article is about the wave front sensor that TNO developed for this mission. This Wave Front Sensor will be used to monitor the wave front errors of the two GAIA telescopes mounted on the GAIA satellite, which may be corrected by a 5-DOF mechanism in orbit. The GAIAWFS will operate over a broad wavelength $(450$ to $900 \mathrm{~nm}$ ) and under cryogenic conditions (130 to $200 \mathrm{~K}$ operation temperature). The WFS uses an all reflective, a-thermal design and uses a Shack-Hartmann micro lens array (MLA)..The focal plane of the WFS is the same as the focal plane of the telescopes. The pattern generated by a star is compared to the pattern of one of the three calibration sources that is included in the WFS.
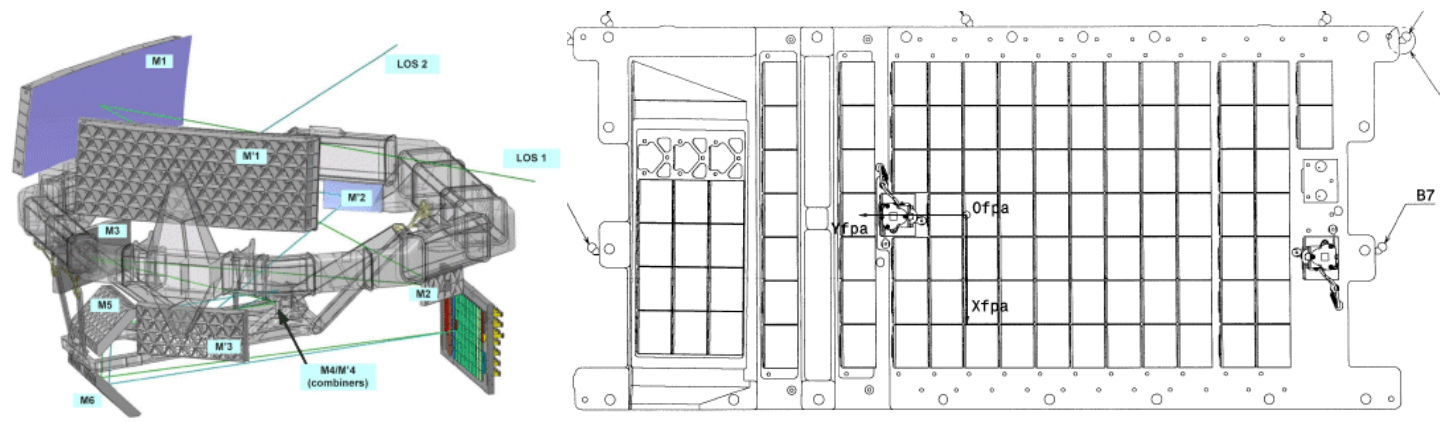

Figure 1-1 A schematic view of the inside of GAIA. Two telescopes (M1) image the stars on a huge focal plane which consists of 108 CCD's. Two CCD's are reserved for wavefront measurements.

*L.L.A.Vosteen@tno.nl; phone +031 152692101; fax +031 152692111; www.tno.nl

Astronomical and Space Optical Systems, edited by Penny G. Warren, Cheryl J. Marshall,

Robert K. Tyson, Michael Lloyd-Hart, James B. Heaney, E. Todd Kvamme, Proc. of SPIE

Vol. $7439,743914 \cdot$ ? 2009 SPIE · CCC code: 0277-786X/09/\$18 - doi: 10.1117/12.825240

Proc. of SPIE Vol. $7439743914-1$ 
This paper presents the robust and lightweight opto-mechanical design that is optimised for launch and cryogenic operation. Furthermore, details on its alignment and commissioning are also presented. The WFS can measure wave front distortions in the order of lambda/1000, and determines the focal plane with an accuracy of $50 \mu \mathrm{m}$

Table 1.The main requirements for the WFS are listed.

- Shack Hartmann measurement method

- Measurement of wave front errors in-orbit and on-ground

- In-orbit calibration by fibre sources

- Entrance FOV: $0.2 \times 0.5 \operatorname{arcmin}^{2}\left(2 \times 5 \mathrm{~mm}^{2}\right)$

- Sampling of wave front: $3 \times 9$ points per pupil

- Uses 1 CCD in the GAIA FPA $\left(59\right.$ x $\left.45 \mathrm{~mm}^{2}\right)$

- Mechanical envelope: approximately $60 \times 60 \times 150 \mathrm{~mm}^{3}$

- Mass: < 500 gram

- Mechanical interface with SiC FPA

- Operation at $160 \mathrm{~K}(130-200 \mathrm{~K})$

- Relative grid distortion $<300 \mathrm{~nm}$

- Magnification: $1 / 6 \pm 1 \%$

- FP accuracy $20 \mu \mathrm{m}$

The interface with $\mathrm{SiC}$, the weight, the low distortion and the operational temperature were the driving force for the opto mechanical design.

\section{WFS PRINCIPLE}

The task of the WFS is to measure the wave front error of the GAIA telescopes on the ground as well as in orbit. To this purpose two small fields at the edge of the astrometric field of view are relayed to a WFS by two fold mirrors in front of the GAIA focal plane (CCD plane), thus producing a sky image at the WFS entrance slit. The WFS entrance slit measures $2 \times 5 \mathrm{~mm}^{2}$, corresponding to the GAIA telescope entrance field of view of $0,2 \times 0,5 \mathrm{arcmin}^{2}$. The optical path of the WFS, extending between the slit and the GAIA focal plane, includes a beam splitter, imaging optics and a microlens array. The imaging optics images the exit apertures of the GAIA telescopes next to each other on the micro-lens array. The micro-lenses each produce an image of the entrance slit on a dedicated CCD at the GAIA focal plane. Thus each micro-lens corresponds to a sample of the telescope pupil and the position of the star image it produces depends on the wave front tilt at that point of the pupil (Shack Hartmann sensor principle). This measurement principle is shown in Figure 2-1

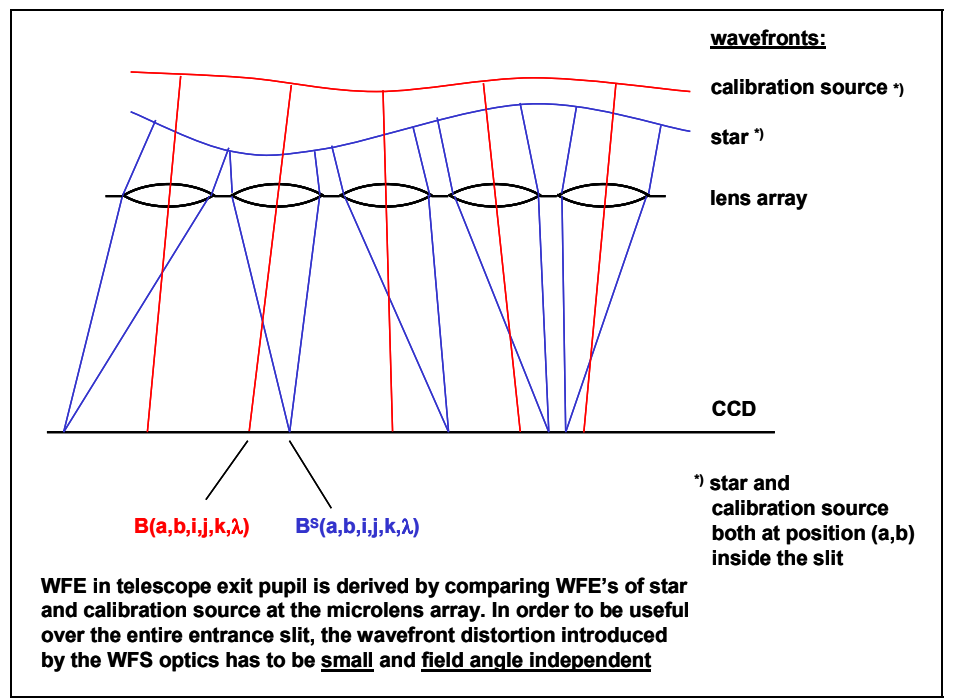

Figure 2-1 The wave front error measurement principle in brief. 


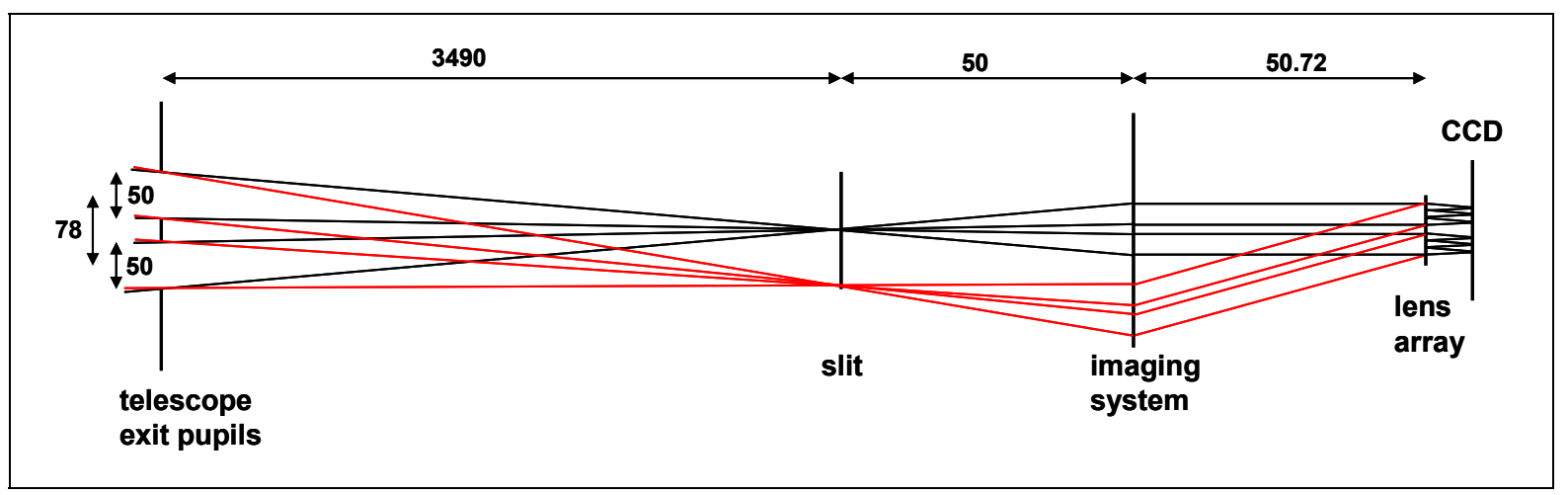

Figure 2-2 Optical chain from telescope exit pupils to GAIA FPA

\section{OPTICAL DESIGN DESCRIPTION OF WFS}

In principle GAIA has two big telescopes, imaging stars with both telescopes on one huge CCD plane. The task of the WFS is to measure the wave front error of the GAIA telescopes on the ground as well as in orbit. To this purpose two small fields at the edge of the astrometric field of view are reserved for wave front measurements. Basically, the WFS relays part of the FoV of GAIA, and while imaging the GAIA exit pupils on the MLA it images the star to the same plane through each lens of the MLA.

Due to the use of the folding mirrors, an intermediate focus is present, allowing the placement of calibration sources virtually in the focal plane, enabling in orbit calibration.

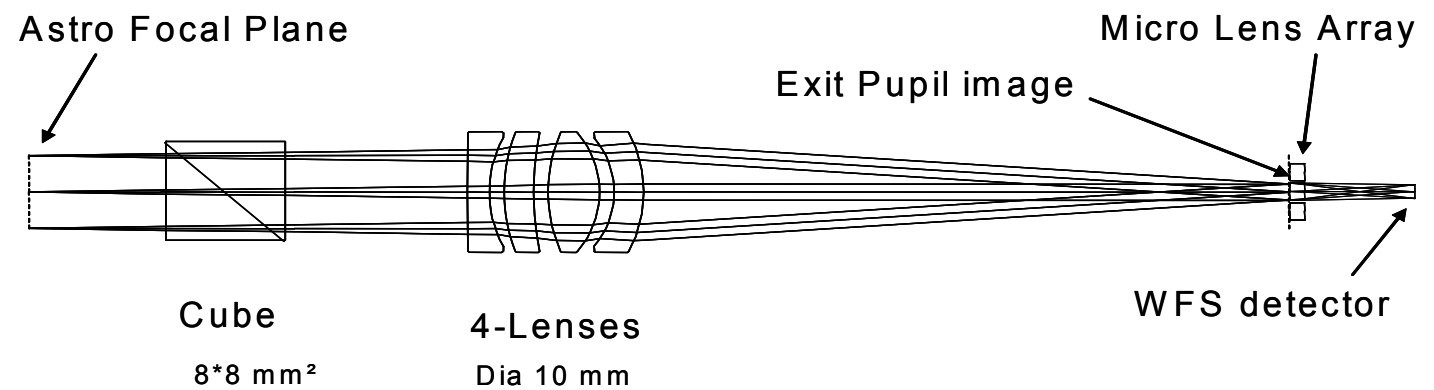

Figure 3.1 Principle design option of GAIA-WFS with quad lens. The beamsplitter cube allows for calibration sources.

\section{DESIGN OPTIONS}

The base design of the WFS is a Shack-Hartman type sensor. The telescope is imaged on the micro lens array, while each micro lens images the star on the focal plane. The first design decision is the selection of the imaging element that images the telescope on the MLA. The second important design decision is the selection of the materials used.

Decisions regarding imaging elements and materials have the largest affect on the design: mass budgets, costs, performance and stability. Furthermore attention has to be paid to the alignment of the system, which is best done at room temperature, while operation is at $160 \mathrm{~K}$.

The other (more detailed) design decisions are: Alignment strategy, Mounts of components, coatings, test facilities.

\subsection{Options for imaging element}

Optical simulations showed that in order to reach all requirements with a refractive element, that element would consist of 4 different types of glass (due to the wavelength range $450-900 \mathrm{~nm}$ ). 
Other simulations showed that the same performance could also be reached with one spherical mirror, with some geometrical constrains (low angle of incidence).

The spherical mirror has an huge benefit in alignment (one element instead of 4), a known temperature behaviour, and can be manufactured with high accuracy. For these reasons, the spherical reflective mirror has been selected for the design of the WFS.

\subsection{MLA optics}

For the microlens array, the wavelength requirement has less influence: the spots will be diffraction limited. The MLA has to be robust to minimize risk during mounting/alignment and cooling down the instrument to 130K. An MLA made from one single piece of fused silica has been chosen, which was then qualified for these environment conditions.

\subsection{Optical design}

The design choice of a focusing mirror instead of lenses leads to the following GAIA-WFS design. A spherical mirror M1, at low angle is used to image the GAIA exit pupil on the MLA (Figure 4.1). The two folding mirrors allow that the focus plane of the MLA is in the same plane as the GAIA telescopes. The Beam splitter just after focus allow the insertion of calibration sources, for the in flight calibration.

The low angle ensures low aberration, and the use of mirrors make the design insensitive to temperature differences.

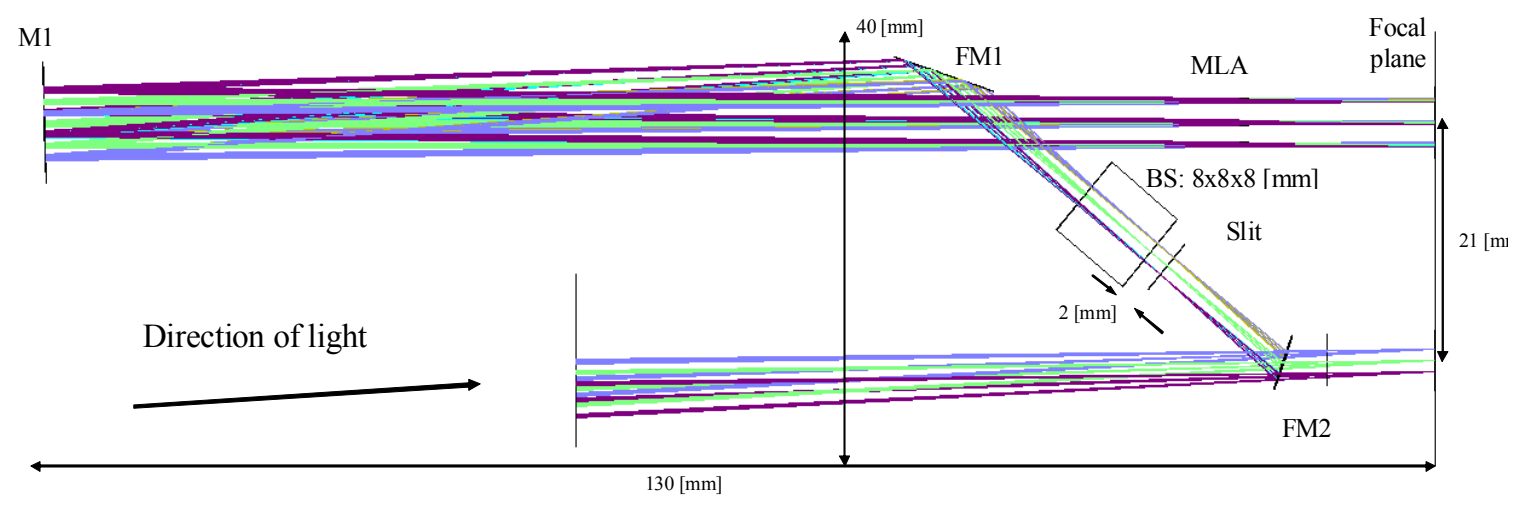

Figure 4.1 The optical design for the GAIA-WFS. M1 images the light from the exit pupils on the MLA. A Beam splitter is used to incorporate calibration sources virtual in the focal plane.

The optical chain after the telescopes exit pupils (3.5 meter to the left) up to the focal plane is shown in Figure 4.1. Two flat folding mirrors (FM2 and FM1) are used to guide the light to a spherical mirror (M1), which images the telescope on the MLA. The beam splitter (BS) behind the slit permits the introduction of 3 calibration sources (in the form of 3 single mode fibres tips) not shown in the figure. Also the general dimensions are displayed: The area span by the mirrors is in the order of 130 by $40 \mathrm{~mm}$.

\subsection{Tolerances}

Detailed analyses of the optical system showed ample integration tolerances (100 um in position accuracy, and $2 \mathrm{mrad}$ in tilt accuracy)

After integration, alignment is performed, increasing for instance the focal length positioning accuracy of the focal plane from about $300 \mathrm{um}$ to accuracy to the required $20 \mathrm{um}$. After the alignment typical stability requirements for the components are $10 \mathrm{um}$ in position, and $50 \mathrm{urad}$ in tilt. These requirements are driven by pupil position budget and magnification factor. 
For the optical performance (differential grid distortion), requirements are set on the surfaces of the mirrors. In the following table the requirements of the optical components are given. Along with the specification of the components the contribution to the differential grid distortion is also noted.

Table 4.1 Shape tolerance specification

\begin{tabular}{|c|c|c|c|c|c|c|c|c|c|}
\hline component: & $\begin{array}{l}\operatorname{Diam}^{1} . \\
\varnothing \\
{[\mathrm{mm}]}\end{array}$ & $\begin{array}{l}\text { radius } \\
{[\mathrm{mm}]}\end{array}$ & $\begin{array}{l}\text { sagitta } \\
\text { error } \backslash \text { tol. } \\
\text { on fringes } \\
\text { A }[\lambda]^{*}\end{array}$ & $\begin{array}{l}\text { differential grid } \\
\text { distortion } \\
\mathrm{x}[\mathrm{nm}]\end{array}$ & $\begin{array}{l}\text { Tolerance on } \\
\text { surface } \\
\text { irregularity } \\
B[\lambda]^{*}\end{array}$ & $\begin{array}{l}\text { differential grid } \\
\text { distortion } \\
\mathrm{x}[\mathrm{nm}]\end{array}$ & $\begin{array}{l}\text { Zernike } \\
\text { terms } \\
14-37 \\
\text { RMS** } \\
{[\mathrm{nm}]}\end{array}$ & $\begin{array}{l}\text { differential grid } \\
\text { distortion } \\
\mathrm{x}[\mathrm{nm}]\end{array}$ & $\begin{array}{l}\text { ISO } 10110 \text { surface form } \\
\text { tolerance } \\
3 / \mathrm{A}(\mathrm{B} / \mathrm{C})\end{array}$ \\
\hline FM2 & $10-4$ & inf. & $\pm 1 / 4$ & 7.5 & $\pm 1 / 20 \backslash \pm 1 / 4$ & $2 \backslash 10$ & \pm 3 & 2.5 & $3 / 0,25(0,05)$ \\
\hline FM1 & $14-4$ & inf. & $\pm 1 / 4$ & 3 & $\pm 1 / 20 \backslash \pm 1 / 4$ & $2 \backslash 10$ & \pm 3 & 2.5 & $3 / 0,25(0,05)$ \\
\hline BS & $\mathrm{X}$ & & & & $\pm 1 / 4$ & 1 & & & \\
\hline $\begin{array}{l}\text { Spherical } \\
\text { mirror } \backslash \mathrm{M} 1\end{array}$ & $16-4$ & 222 & $\begin{array}{l} \pm 1 / 4 \\
\text { radius } \pm 0 \\
2 \%\end{array}$ & $\begin{array}{l}5 \\
12\end{array}$ & $\pm 1 / 15$ & 5 & $\pm 3 \backslash \pm 6$ & $30 \backslash 80$ & $3 / 0,25(0,07)$ \\
\hline Lens array & $X$ & & $\begin{array}{l}\text { radius } \pm 3 \\
\% \\
\end{array}$ & 2.5 & & & & & \\
\hline & & & & & & & & & \\
\hline
\end{tabular}

1: total diameter - mounting area $=$ effective optical diameter $(10-4$ comes to $6 \mathrm{~mm}$ effective optical diameter $)$

$* \lambda=630[\mathrm{~nm}]$ (double pass Newton's rings test) over total diameter

** square root of the sum of the squares of the coefficients. Test measurements performed on a high quality flat mirrors resulted in values between 2 and $3 \mathrm{~nm}$ rms, justifying these values as requirement.

The flat folding mirrors (FM1 and FM2) both have the requirement of surface irregularity of $\lambda / 20$. This results in a $2 \mathrm{~nm}$ differential grid distortion. For information, in the table is also noted the effect if the mirrors are $\lambda / 4$ : the contribution to the differential grid distortion is then increases to $10 \mathrm{~nm}$. From the table is concluded that the shape of the spherical mirror is the parameter that is the most critical one for achieving the required performance, its contribution is $30 \mathrm{~nm}$ differential grid distortion.

\section{MECHANICAL DESIGN}

Mechanically the WFS design consists of an Invar interface structure and an optical housing, an Invar tube to mount the optical components on, see Figure 5.1. The Invar interface has flexures to compensate for thermal expansion differences between the $\mathrm{SiC}$ support structure and the OMA. The three footings of the Invar interface are bolted to the SiC support. The WFS will be aligned to the CCD plane using Invar shims under the 3 footings. For alignment 2 reflective references are provided (X-Y, X-Z)

The optical column is mounted on the Invar interface structure. The optical parts ( 3 mirrors, Beam splitter, MLA and a Ferrule) are mounted on the outside of the optical housing. Optical components including the ferrule are readily accessible during mounting and alignment, because of their exterior attachment to the housing (Figure 5.3). 


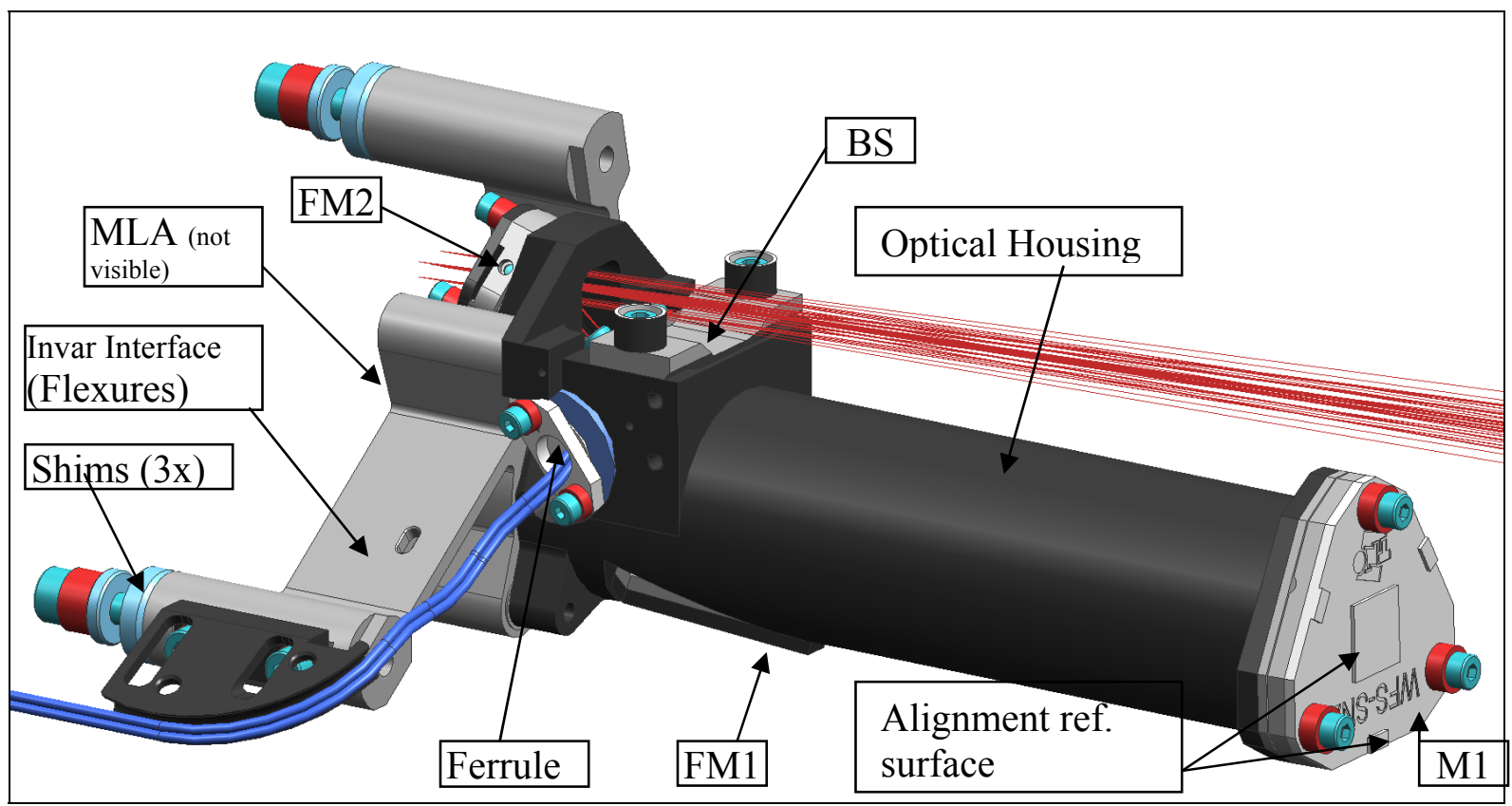

Figure 5.1 Overview OMA design, indicating the two folding mirrors (FM1 and FM2), the spherical mirror (M1), the beam splitter (BS), the micro lens array (MLA), the ferule which consists of 3 monomode fibre tips as calibration sources and the structure.

Figure 5.2 shows the mounting of folding mirror 2 (FM2). All mirrors are mounted according to this principle. The Mirror assembly consists of an optical mount, mirror cover and a mirror. The mirror is made of fused Silica and the other parts of Invar (Annealed M93). This material combination gives the best CTE match to invar. In the mirror mount 3 tangential leaf springs are made by electro-erosive metal removing. The Mirror is centred by these leaf springs and bonded to them with RTV 566. Bread board tests have proven that this construction leads to high thermal stabilities.

The shapes of the mirror and mount are given by the envelope, optical path, alignment and (thermal) stability. 


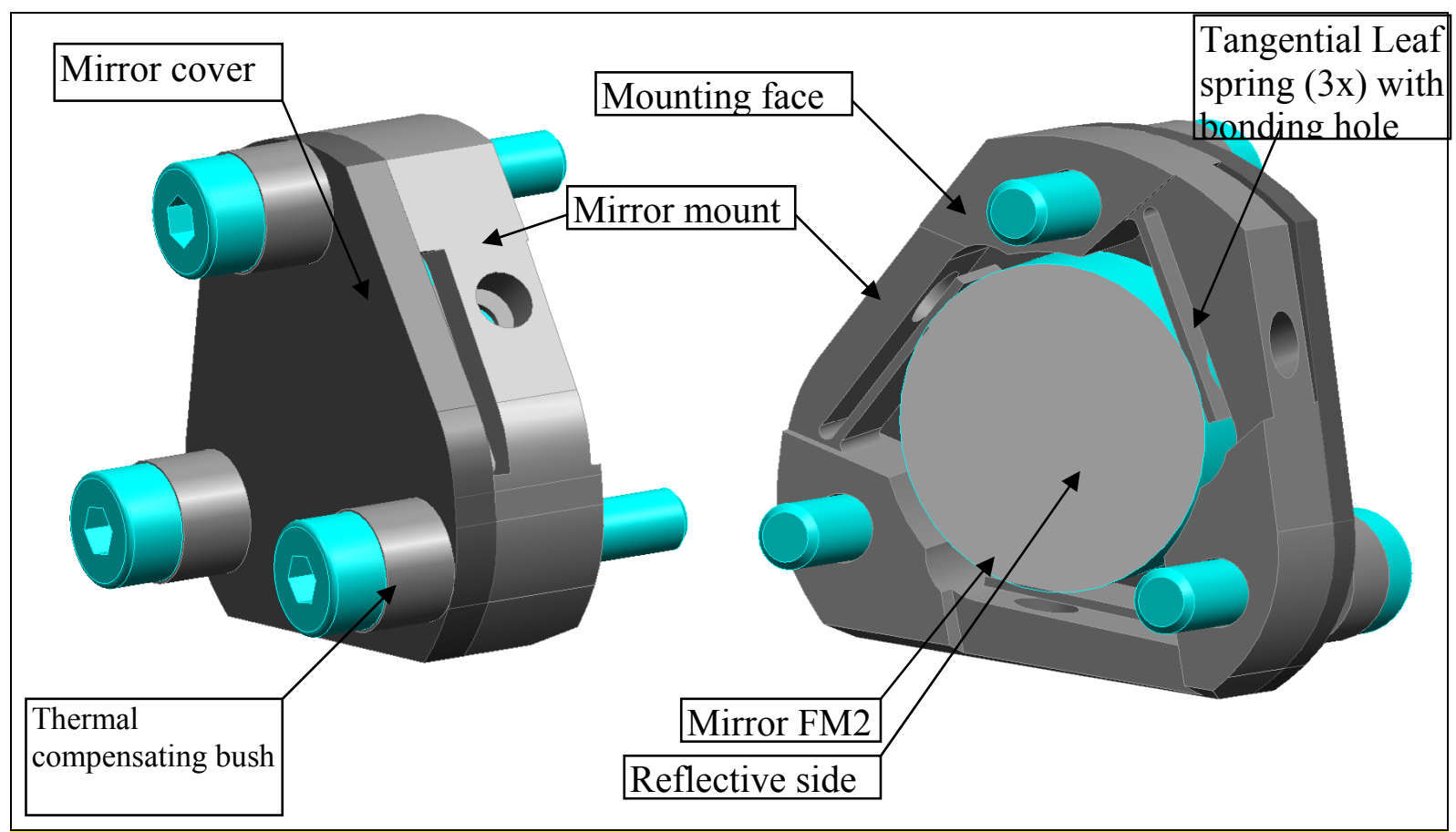

Figure 5.2: Mirror Assy FM2. The invar mount consists of three leave springs, ensuring low (thermal) forces on the mirror.

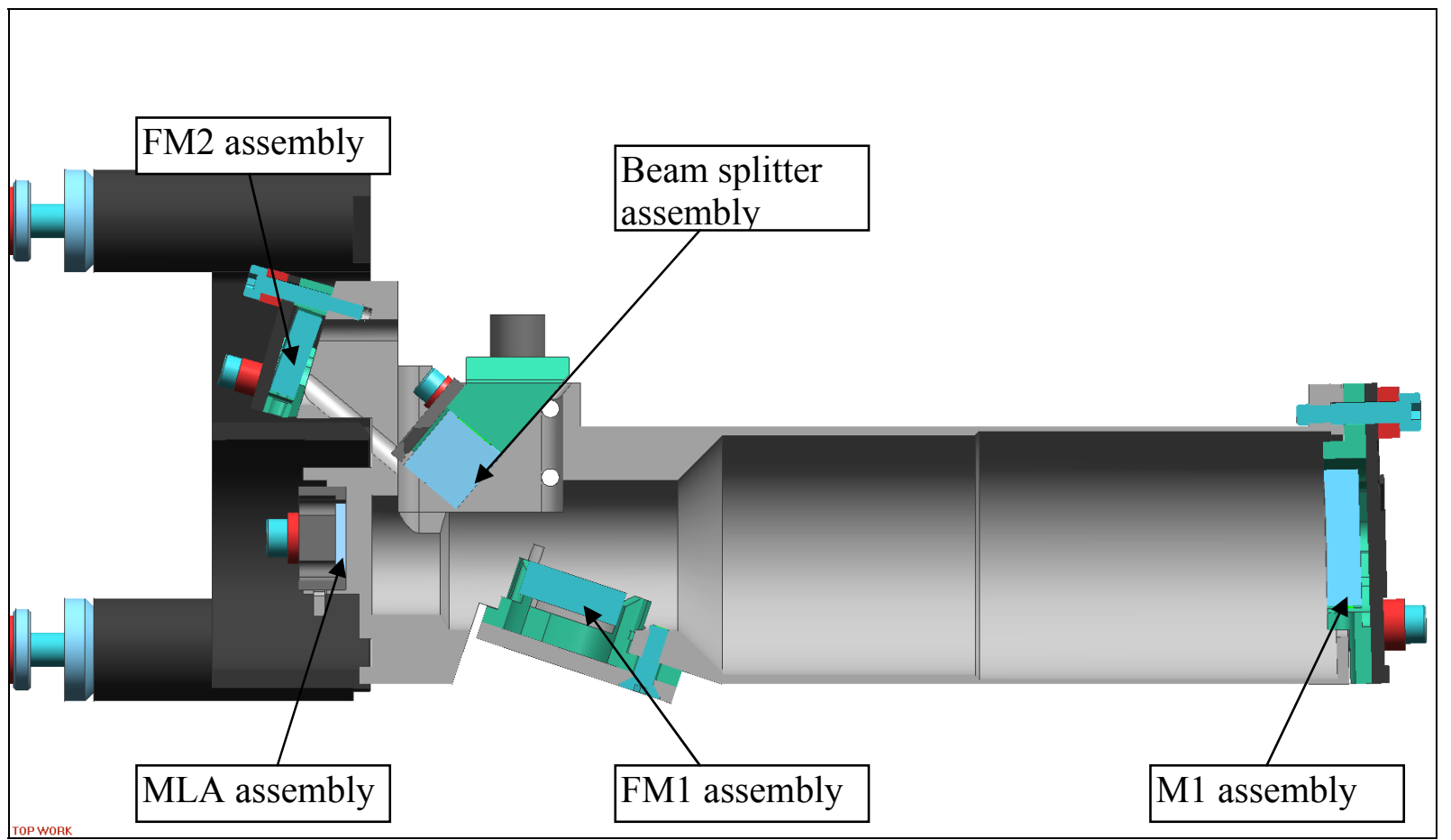

Figure 5.3: Optical components mounted to the Optical housing (section view) 


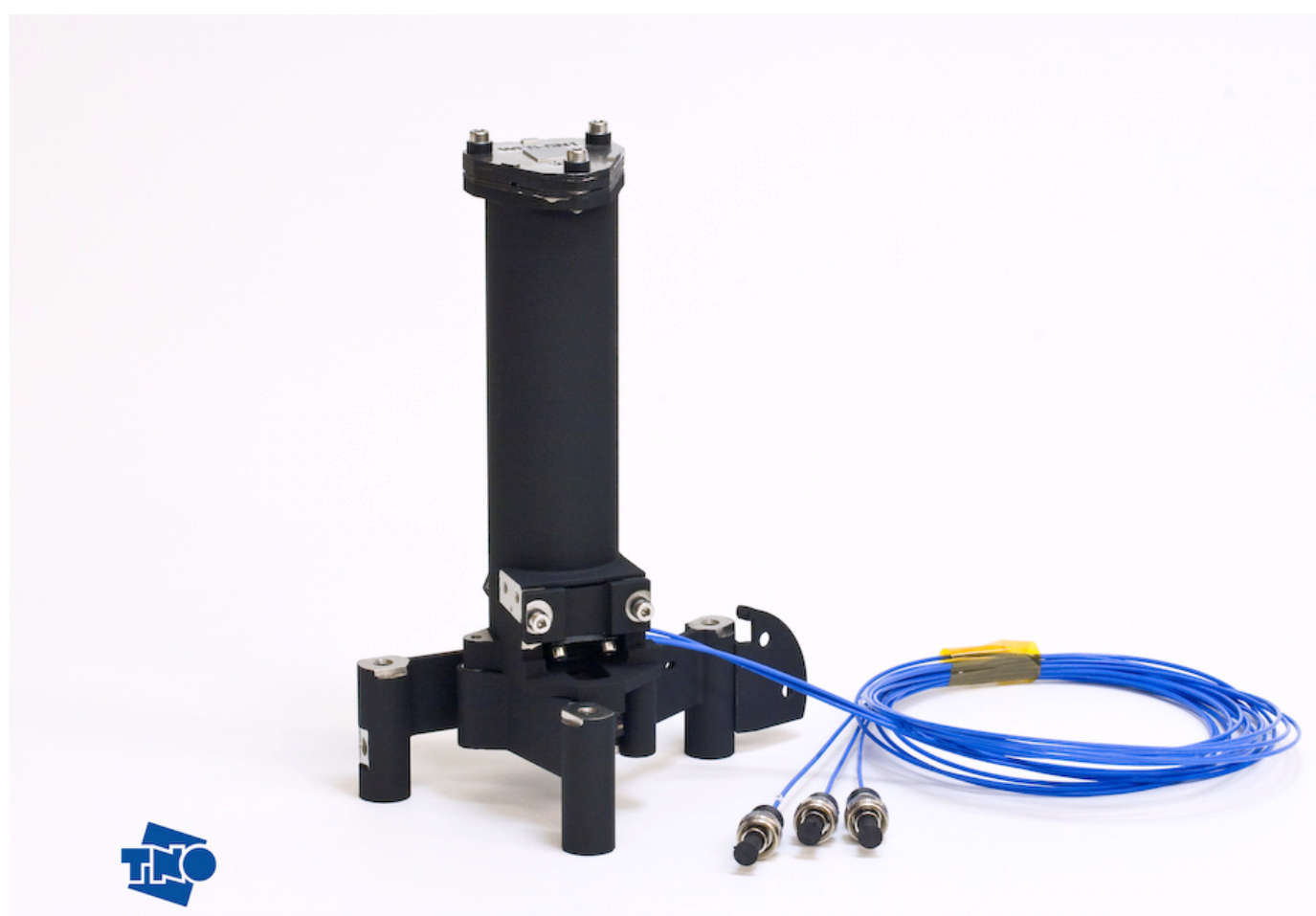

Figure 5.4: The actual hardware. The WFS is made black for stray light reasons. The flexures can be seen at the bottom, and the blue calibration fibres are shown. The all invar WFS is about $150 \mathrm{~mm}$ high, and weighs less then 500 grams.

\section{ON GROUND SUPPORT EQUIPMENT}

The purpose of the on ground support equipment (OGSE) is to demonstrate the performance of the qualification and flight hardware. The main functions are listed:

Simulate the GAIA telescopes.

Simulate a star inside the FoV of the telescopes

Simulate the environment (130k to $200 \mathrm{~K}$, operational at $160 \mathrm{~K}$ )

Measure the performance of the WFS with sufficient resolution 


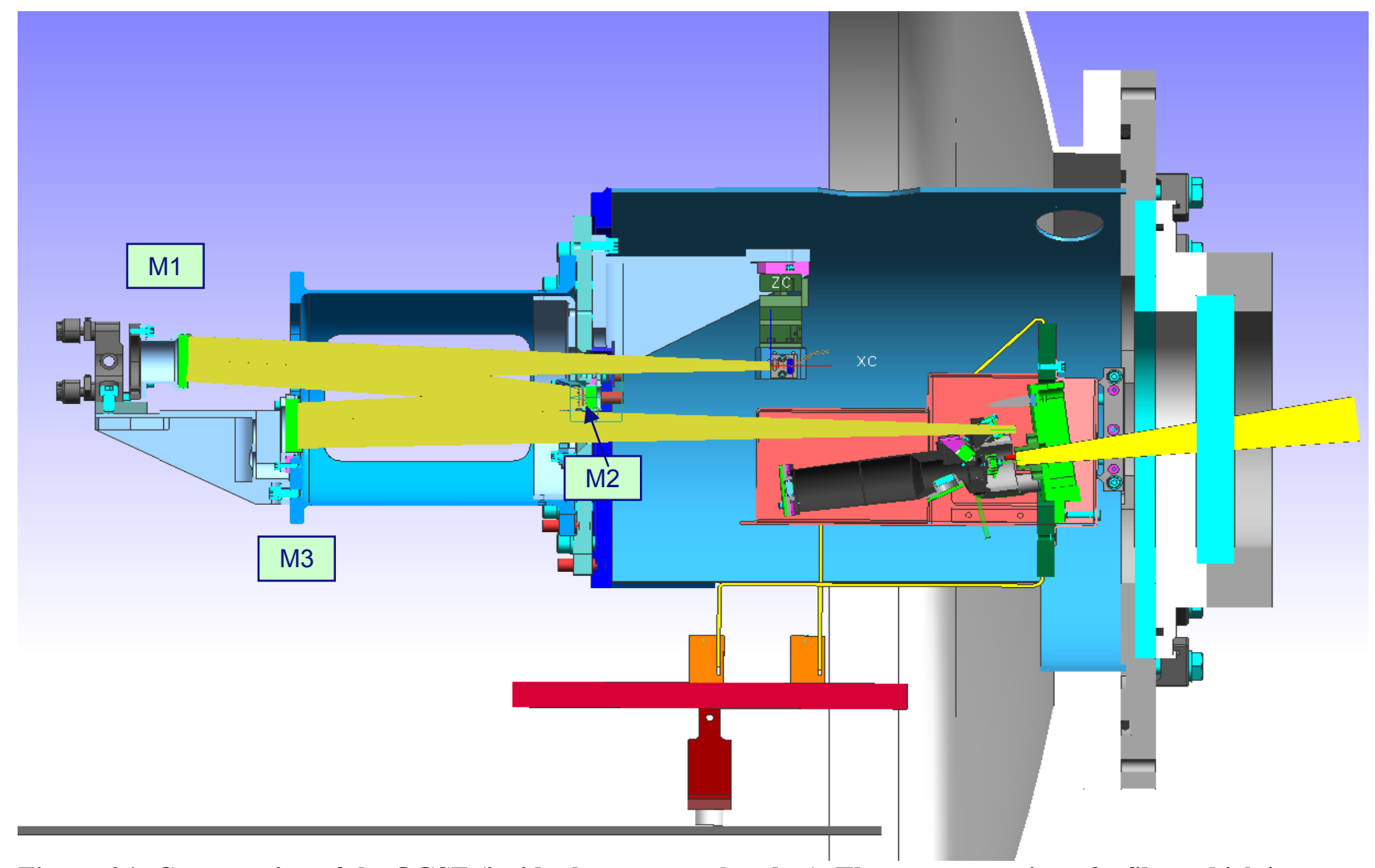

Figure 6.1: Cross section of the OGSE (inside the vacuum chamber). The system consists of a fibre which is mounted on an xyz-translation stage. An a-symmetrical Offner system (M1, M2 and M3) image the fibre on the focal plane. The WFS is mounted inside a cold box. Only the box with the WFS is cooled.

\section{PERFORMANCE}

The performance of the WFS for different environmental conditions has been determined. The differential grid distortion over one line-scan in the field of view has also been determined.

The performance is noted for the grid consisting of all spots, and for $89 \%$ of the spots (omitting the extreme spots).

Atmospheric distortion, vibration, random distortion due to the OGSE, in addition to the inherent WFS performance, can be expected to influence the expected results.

During the test campaign of the WFS, at different moments a series of optical measurements have been performed to determine the optical performance. In the test campaign these series are numbered from 1 to 13 . For this article only 1,8 , 12 and 13 are reported. These represent the conditions from ambient (1), 160K (8), ambient (12) and after vibration (13). These points are chronological in time. For these points one scan in the FoV is presented: a star travelling from location G1 to G2 to G3, and the distortion is compared to a star at location E2. (position of calibration star) This covers the full field in the y direction.

In Figure 7.1 a difference can be seen between the left and right pupil. This difference has been identified as a contribution from the OGSE: For the measurement, the alignment has been performed on the left pupil, which results in a lower performance for the other (not so well aligned) pupil. For real operation both pupil will have the same (high) performance. 


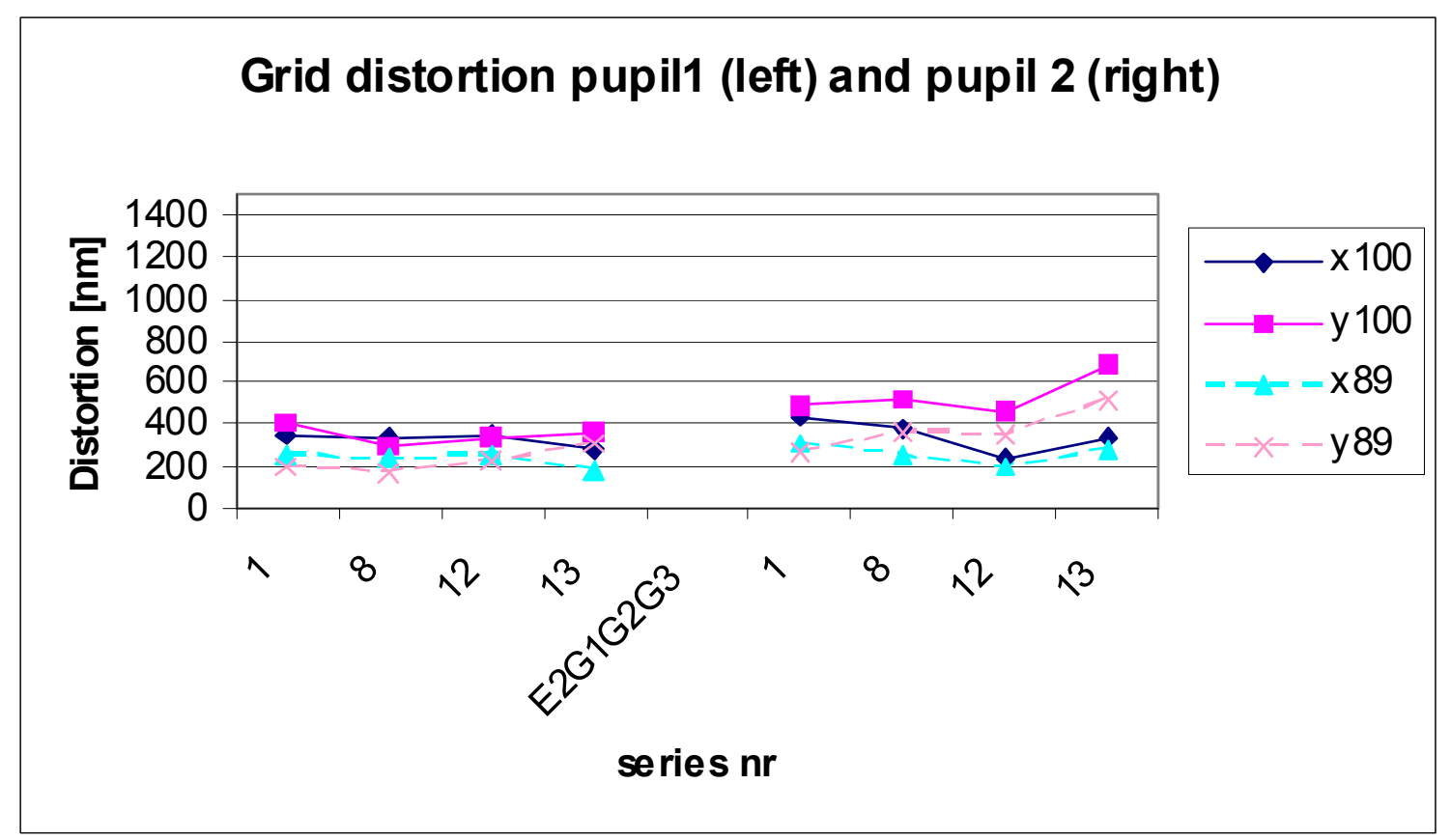

Figure 7.1: The differential grid distortion for different conditions. The performance is measured at ambient, vacuum, at $160 \mathrm{~K}$ and after vibration. The left curve is given for pupil 1 , the right curve is for pupil 2 .

\section{CONCLUSION}

The GAIA wavefront sensor has been designed, build and tested. The WFS Qualification model has passed the vibration and thermal cycle tests. The optical performance is $300 \mathrm{~nm}$ differential grid distortion, which equals $0.7 \mathrm{~nm} \mathrm{rms}$ wavefront error.

The requirement for absolute focal plane accuracy of $20 \mathrm{um}$ is very difficult to prove due to measurement limitations. The accuracy of $50 \mathrm{um}$ focal plane comes from the measurement uncertainty of the different contributors.

The coating qualification program is still ongoing, the final radiation tests will be done monetary.

\section{REFERENCES}

[1] ESA Science \& Technology http://sci.esa.int/GAIA 